\title{
Rick ANDERSON, Scholarly communication. What everyone needs to know
}

Ivan Jaffrin

\section{(2) OpenEdition}

1 Journals

Édition électronique

URL : http://journals.openedition.org/ress/6117

DOI : 10.4000/ress.6117

ISBN : $1663-4446$

ISSN : $1663-4446$

Éditeur

Librairie Droz

Édition imprimée

Date de publication : 6 juillet 2020

Pagination : 297-302

ISSN : 0048-8046

Référence électronique

Ivan Jaffrin, «Rick ANDERSON, Scholarly communication. What everyone needs to know ${ }^{\circledR}$ ", Revue européenne des sciences sociales [En ligne], 58-1 | 2020, mis en ligne le 06 juillet 2020, consulté le 01 décembre 2020. URL : http://journals.openedition.org/ress/6117; DOl : https://doi.org/10.4000/ress. 6117

Ce document a été généré automatiquement le 1 décembre 2020.

(c) Librairie Droz 


\title{
Rick ANDERSON, Scholarly communication. What everyone needs to know
}

\author{
Ivan Jaffrin
}

\section{RÉFÉRENCE}

Rick ANDERSON, 2018, Scholarly communication. What everyone needs to know ${ }^{\circ}$ New York, Oxford University Press, $280 \mathrm{p}$.

1 Rick Anderson est un personnage bien connu des professionnels de l'information et de la communication scientifiques. Lui-même doyen associé (associate dean) à la Willard Marriott Library (Université de Utah), il a occupé durant toute sa carrière différents postes dans le secteur des bibliothèques universitaires, en charge de l'acquisition des ressources et de la gestion des collections, tout en assurant des activités de conseil auprès de grands éditeurs scientifiques. Il doit sa notoriété publique à ses qualités reconnues d'expert en matière de "communication scientifique", à travers de multiples conférences et analyses, ainsi qu'à ses contributions régulières au blog professionnel Scholarly Kitchen, lancé en 2008 à l'initiative de la Society for Scholarly Publishing - dont Rick Anderson est élu président en 2015.

Il était donc l'auteur tout indiqué pour cet ouvrage, Scholarly communication, paru aux presses de l'université d'oxford, dont l'objectif assigné par la collection qui l'accueille "What everyone needs to know» - vise à présenter un panorama assez complet des acteurs et enjeux du secteur d'une manière à la fois synthétique et objective. Telle est d'ailleurs l'ambition affichée dès l'introduction par l'auteur qui, tout en rappelant les multiples controverses qui agitent actuellement le monde de la communication scientifique, entend bien prendre soin d'éviter les partis pris trop marqués. Cependant, nous ne ferons pas mystère du fait que Rick Anderson occupe une position particulière dans le débat autour du libre accès aux ressources scientifiques (open access) qui le place 
davantage du côté des tenants du « réalisme » que des « idéalistes »- si l'on veut bien se satisfaire provisoirement d'une opposition aussi binaire que contestable (voir Anderson, 2014, "Is rational discussion of open access possible? », Insights: the UKSG journal, 27-2, p. 171-180 [en ligne] : <https://insights.uksg.org/articles/10.1629/2048-7754.162/ $>$ ). Et, de fait, il s'agit pour Rick Anderson, tout au long des 14 chapitres qui rythment cet ouvrage, d'examiner en détail les contours d'un objet instable et en constante redéfinition sans faire fi des rapports de force ni des contraintes qui pèsent sur cet objet - la communication scientifique - et non pas d'asséner les vertus d'un modèle alternatif qui peine encore à voir le jour.

3 Disons-le d'emblée, le lecteur déjà informé des enjeux de la communication scientifique n'apprendra rien de fondamentalement nouveau à la lecture de cet ouvrage. Cependant, tout l'intérêt de l'entreprise menée par l'auteur réside moins dans le caractère exhaustif de l'exposé que dans l'horizon sous lequel il se déploie. Prenant soin d'examiner tous les aspects de la communication scientifique à l'aune des transformations en cascade que lui fait subir le développement du numérique - dont l'enjeu de l'open access est directement dérivé -, l'auteur s'évertue à saisir la dynamique de tout un secteur, de manière à en apprécier au plus près les mutations en cours.

Pour ce faire, il convient naturellement d'abord de délimiter le périmètre de ce dont on parle et de s'accorder sur quelques définitions préalables. Tel est l'objet du tout premier chapitre (Chap. 1. "Définition and History»), qui commence par poser que par "scholarly communication", il convient d'entendre toute forme de "research-based communication » (p.13), à savoir, en premier lieu, les articles de recherche parus dans les quelques 30000 revues périodiques à comité de rédaction répertoriées dans le monde (p.14), mais également les monographies, rapports de recherche, versions d'articles préliminaires, thèses, supports de conférences, billets de blog, etc. (p. 5 et suiv.). Le terme "scholarly» réfère quant à lui plus précisément au milieu académique des chercheurs et collègues - les pairs - mais également aux étudiants, doctorants ou post-doctorants, et ce dans un contexte institutionnel marqué par l'obligation faite aux chercheurs de publier pour s'assurer une position permanente («tenure track» - p. 15).

De ce tour d'horizon rapide, l'auteur en déduit un terme capital pour la suite de l'exposé: la communication scientifique en tant qu'elle donne à voir un réseau d'organisations et d'acteurs engagés dans la production d'un produit spécifique au travers une logique de compétition et de coopération est fondamentalement un « écosystème » (p.17). C'est à l'inspection de cet éco-système que s'attachent les 7 chapitres suivants. Passant tour à tour en revue les différents acteurs de la communication scientifiques (Chap. $2:$ «Who Are the Scholars and Why They do Communicate?») à l'aune d'un marché de la publication scientifique pesant 26,2 milliards de dollars rien que dans les sciences techniques et médicales (à comparer avec les 5 milliards estimés pour les sciences humaines et sociales [p. 52] - Chap. $3:$ «What Does the Scholarly Communication Marketplace Look Like?»), l'auteur en vient à observer concrètement leur manière de coopérer et de se répartir les tâches et fonctions éditoriales (Chap. $4:$ «What Is Scholarly Publishing and How Does It Work ?»), tout en rappelant l'importance cruciale de la législation en matière de droit d'auteur dans l'équilibre général de cet éco-système (Chap. 5: "What Is the Role of Copyright?»). L'auteur se focalise ensuite sur le rôle spécifique de deux acteurs publics majeurs qu'il connaît bien (Chap. $6:$ « What Is the Role of Library» et Chap. $7:$ "The Role of University Press »), puis parachève provisoirement 
son exposé avec un aperçu des nouveaux entrants dans le secteur que sont les bibliothèques numériques (Chap. 9 : «Google Books and HathiTrust »).

Pour être assez complet, l'examen de cet éco-système complexe n'en donne pas moins une vision globalement très anglo-américaine qui n'est pas transposable en l'état au monde francophone, comme en témoigne la section dédiée à l'organisation du champ académique entre college et university, (p. 30 et suiv.) ou la définition du copyright selon la loi américaine et le régime de fair use (p. 91), ou bien encore l'importance des sociétés savantes (p. 80-81) dans l'organisation de communautés disciplinaires et le caractère incontournable des presses universitaires. Un certain nombre de faits prégnants émergent cependant qui tendent à structurer l'analyse d'une manière qui reste pertinente pour le lecteur francophone, à savoir 1. la compétition pour les places dans un régime où la publication est l'objectif primordial (" publish or perish»-p. 15 et 49-50) ; 2. la distinction remarquable entre sciences techniques et médicales (STM) et sciences humaines et sociales (SHS) - laquelle fait d'ailleurs l'objet d'un chapitre à part, tant elle s'avère particulièrement décisive dans le contexte numérique (voir p. 207-208 et Chap. 9 "Needs and Practices in STM and HSS ») -, à travers notamment l'importance encore avérée de la monographie dans ce dernier secteur (p. 6, 22 et 177); 3. le rôle crucial exercé dans tous les cas par les pairs dans l'exercice de l'évaluation scientifique (peer-review - p. 64); et 4. le fait que l'organisation de la publication scientifique reste largement tributaire d'éditeurs, privés ou publics, non dépourvus d'intérêts économiques et commerciaux (p. 75, 81-82 et 151). Il apparait ainsi que la publication en revue - et dans les revues à plus fort impact -, demeure en général le graal dans l'avancement académique des chercheurs, plaçant de fait les éditeurs en situation de monopole (p.53), au détriment le plus souvent des ouvrages qui tendent à être marginalisés dans la communication scientifique (p. 159, 226-228 et 250-251).

7 Mais s'il est une ambition qui nous paraît motiver toute l'entreprise de l'auteur et soustendre son propos tout au long de l'exposé, elle procède plus fondamentalement de l'attention minutieuse qu'il apporte aux tensions qu'exerce l'irruption du numérique sur chacun des acteurs de l'éco-système traditionnel de la communication scientifique. Le numérique, en permettant la dissémination massive et instantanée des contenus à un coût marginal nul, ou quasi-nul (p. 22), oblige à un travail collectif de re-définition du cahier des charges de la communication scientifique émancipée des contraintes physiques en termes de format et de support (p. 29 et 199-200). L'avènement du numérique tend alors à faire de l'article de recherche l'unité de base de la communication scientifique en lieu et place de la revue, dont le format autant que le rythme de publication résultaient de contraintes qui n'ont plus lieu d'être en régime numérique (p. 20 et 249). La communication scientifique s'en trouve ainsi libérée des entraves du monde physique, la diffusion s'effectuant principalement par le réseau avec une efficacité jamais atteinte par la publication papier. Ce faisant, l'indexation des contenus sur le Web modifie fondamentalement leur «discoverability» (p. 62 et 119) et transforment les usages de consultation, à travers la recherche plein-texte par exemple. Il en résulte l'émergence de nouveaux services et de nouvelles fonctions qui bouleversent le champ de compétence des acteurs traditionnels de la publication scientifique, mais également de nouveaux modèles économiques fondés sur le libre accès. L'émergence de plate-formes de publication purement numériques en libre accès, avec le cas des megajournals (p. 212-213 et 250), en est un exemple probant ainsi que le développement des réseaux sociaux académiques (p. 256-257). En première ligne face à ses mutations, les bibliothécaires voient quant à eux les fondamentaux de leur 
métier changer radicalement (p. 232-233), voire tendent à s'imposer eux-mêmes progressivement comme éditeurs de contenus (p.134 et suiv.), à travers le développement des répertoires institutionnels (p. 215). Il n'est pas jusqu'au principe de la collection qui ne se voit remis en cause par le numérique à la faveur de modèles d'acquisition alternatifs tirés par la demande (demand-driven acqusition) et non plus par une logique d'offre (p.121 et suiv). Enfin, ce basculement vers ce qui pourrait constituer à terme un nouvel éco-système numérique s'accompagne de la promotion d'alternatives au système légal de copyright, visant à optimiser le potentiel de diffusion des contenus en ligne, avec le développement des Creativ Commons (copyleft - p. 104 et suiv.).

8 Pour autant, l'auteur ne se résout pas à sonner le glas de la communication scientifique traditionnelle. C'est ainsi tout l'intérêt de son exposé que de montrer que le passage d'un d'un éco-système papier à un autre, numérique, ne va pas sans heurts. Loin de s'ajuster les unes aux autres de manière synchrone et simultanée, les différentes composantes de l'éco-système apparaissent au contraire comme travaillées par des tendances parfois contradictoires, sujettes à l'inertie ou bien à des contraintes techniques, économiques, légales ou institutionnelles, qui sont autant de frictions tendant à retarder indéfiniment l'avènement d'un nouvel équilibre "purement » numérique - lequel reste in fine l'objet d'intenses spéculations.

Abstraction faite des différences - essentielles - qui subsistent selon les disciplines et principalement entre les SHS et les STM, c'est l'idée même d'un basculement vers un modèle alternatif de communication scientifique faisant table rase de l'éco-système précédent qui est relativisé par l'auteur. Ainsi, loin d'être supplantée par les outils numériques de communication, l'étape de la publication en revue s'avère conserver pleinement sa fonction traditionnelle de validation et de consécration et se voit même investie en retour de la fonction supplémentaire d'archivage de la version faisant autorité (version of record), dans un contexte où tendent à proliférer différentes versions d'un même article à différents stades d'écritures et de contrôle (p. 42-44). Qui plus est, dans bien des cas, le numérique n'a pas supplanté le support imprimé, à l'endroit duquel subsiste une demande difficilement réductible (p.29), mais s'ajoute à lui, redoublant le processus de publication de tâches complexes qui, loin de diminuer les coûts de production, tendent au contraire à les renchérir (p. 29). Non seulement la logique de "plus-value éditoriale» (p. 59 et suiv.) n'a pas disparu, mais elle s'avère encore plus décisive en contexte numérique où archivage, indexation et traçabilité (p.30) deviennent des enjeux cruciaux, comme en témoigne le développement de mesures d'impact des articles en temps réel (p. 187-189 et 237), supposées remplacer à terme le très contestable facteur d'impact (Chap. 10: "Metrics and Altmetrics»), ainsi que, plus généralement, le rôle crucial joué dans l'ensemble de l'éco-système numérique par les métadonnées (Chap 11. " Metadata and Why It Matters»).

Si ces deux derniers chapitres tendent à confirmer la portée considérable de la transformation numérique en cours, cette dernière semble cependant n'avoir pas affecté sensiblement l'une des fonctions-clés de la communication scientifique, à savoir l'évaluation par les pairs. En dépit de tentatives encore infructueuses (open peer-review, post-peer review - p. 65) d'en renouveler de fond en comble l'organisation - souvent jugée sous-optimale (p.66-67 et 235) -, elle continue en effet à jouer un rôle incontournable sous une forme somme toute très traditionnelle. De fait, quoique l'évaluation soit en grande partie exercée bénévolement par les chercheurs eux- 
mêmes, comme l'auteur n'omet pas de le préciser (p.47-48 et 201), la gestion du processus éditorial de l'évaluation à la sélection demeure l'une des fonctions les plus fastidieuses et coûteuses de l'éco-système à la charge des revues (p. 66), lesquelles - il faut s'en étonner - n'ont pas encore trouvé à être remplacées par un système numérique plus performant et sont donc toujours le principal canal de la communication scientifique. C'en est au point que l'auteur est fondé à s'interroger sur la portée de la révolution numérique en cours en ces termes : «It has been simultaneously hugely disruptive and not disruptive at all » (p. 20-21).

11 C'est justement sur la base de ce constat en demi-teinte, posé très tôt dans l'exposé, que l'auteur entreprend pour finir, dans une série de 3 chapitres conclusifs, d'appréhender à nouveaux frais les évolutions présentes et futures de la communication scientifique (Chap. 12. "Open Access: Opportunities and Challenges »; Chap. 14. "The Future of Scholarly Communication »), à l'aune des controverses auxquelles elles donnent lieu (Chap. 13: "Problems and Controversies in Scholarly Communication»). C'est sans doute le terrain sur lequel l'auteur était le plus attendu, mais également celui sur lequel les contraintes d'un tel ouvrage de synthèse se font le plus sentir. De fait, les enjeux sont d'une redoutable complexité et l'auteur ne fait ici que les efflorer, non sans un effort louable d'objectivité et de pédagogie ne pouvant que profiter au lecteur non-initié. À défaut de pouvoir nous faire ici l'écho de l'ensemble des sujets abordés par l'auteur, l'on remarquera cependant que l'une des questions lancinantes au cœur des controverses en cours (serial crisis [p. 225], big deals [p. 228], predatories journals [p. 238 et suiv.], APC et hybrid journals [p. 205-206], etc.) repose en dernière analyse sur la mise en question du rôle des éditeurs dans l'ensemble de l'éco-système et de la justification du coût de l'accès à l'information scientifique, dès lors que celle-ci est produite en numérique et reproductible à moindre coût (p. 78). À cet égard, l'avènement d'un nouvel éco-système numérique, articulé autour du libre accès aux ressources scientifiques, manque d'autant moins d'avocats qu'il consommerait définitivement la ruine du modèle économique classique de la souscription et mettrait ainsi un terme aux excès du «capitalisme de rente » auquel il donne lieu avec le monopole de quelques très grands éditeurs commerciaux fixant des prix quasi prohibitifs pour l'accès à leurs bases de données. Or, comme le remarque l'auteur: "It may be that we actually no longer need publishers, but that neither scholarly and scientific authors nor paying readers have yet caught up to this reality» (p. 79).

Cette réponse non dépourvue de malice traduit finalement bien l'esprit pragmatique de l'ouvrage, l'auteur se refusant à spéculer davantage sur l'avenir numérique de la communication scientifique pour en rester sur le terrain des faits, quand bien même ils décevraient les attentes. Tel était son cahier des charges. Telle est également sa limite. Car au fond, ce qui se dessine au fil de l'ouvrage n'encourage pas forcément une lecture en termes d'émancipation de la communication scientifique des formats et contraintes d'un modèle économique et capitalistique rendu obsolète par le numérique, conformément aux vœux des partisans les plus fervents du libre accès. Loin de réaliser toutes les promesses de collaborativité et de transparence portées par les digital humanities (p. 257 et suiv.), le libre accès apparaît même possiblement comme l'un des leviers d'une "plate-formisation » de la science, articulée sur la capitalisation des données, susceptible de traduire un nouvel équilibre de l'éco-système de la communication scientifique dans l'environnement numérique, très éloigné de l'idéal d'ouverture et d'échange à l'origine du mouvement. Non seulement le libre accès s'appuie désormais sur des modèles économiques éprouvés (p. 205), mais rien n'indique 
qu'il participe in fine, et à lui seul, d'un affranchissement de la recherche et des chercheurs des intérêts économiques, tant il s'est manifestement imposé lui-même comme l'un des principaux paramètres actuels de la course à la publication, sans en modifier aucunement les règles du jeu ni en diminuer la cadence. Bien au contraire.

\section{AUTEURS}

\section{IVAN JAFFRIN}

Revue européenne des sciences sociales, Librairie Droz 